\title{
Measuring accessibility to hospitals by public transport: An assessment of eight Canadian metropolitan regions
}

\author{
Geneviève Boisjoly \\ Department of Civil, Geological and Mining Engineering, Polytechnique Montréal \\ gboisjoly@polymtl.ca \\ Robbin Deboosere \\ School of Urban Planning, McGill University \\ robbin.deboosere@mail.mcgill.ca
}

\section{Rania Wasfi}

Centre de Recherche du CHUM, Université de Montréal

rania.wasfi@crchum.qc.ca

\section{Heather Orpana}

Centre for Surveillance and Applied Research, Public Health Agency of Canada

heather.orpana@canada.ca

\section{Kevin Manaugh}

Department of Geography, McGill University

kevin.manaugh@mcgill.ca

\section{Ron Buliung}

Department of Geography and Planning, University of Toronto ron.buliung@utoronto.ca

\author{
Ahmed El-Geneidy \\ School of Urban Planning, McGill University \\ ahmed.elgeneidy@mcgill.ca
}

\section{ACKNOWLEDGEMENTS}

This study was conducted with the financial support of the Canadian Social Sciences and Humanities Research Council.

For Citation Please use: Boisjoly, G., Deboosere, R., Wasfi, R., Orpana, H., Manaugh, K., Buliung, R. \& El-Geneidy, A. (2020). Measuring accessibility to hospitals by public transport: An assessment of eight Canadian metropolitan regions. Transport and Health, 18, 1-14. 


\section{ABSTRACT}

\section{Introduction:}

3 The ability to access healthcare services has long been considered a 'right' by Canadian citizens and is

4 protected as such under the Canada Health Act. However, socio-spatial factors can limit access to healthcare

5 services, especially for vulnerable populations. This paper aims to quantify the spatial accessibility to

6 healthcare services by public transport across eight major Canadian metropolitan areas and compare

7 accessibility to healthcare across vulnerable population groups. This is the first study, to the authors'

8 knowledge, to conduct an assessment of accessibility to hospitals by public transport across multiple

9 metropolitan regions in Canada, thereby allowing to identify common trends, challenges, as well as

10 knowledge gaps.

\section{Methods:}

12 Spatial accessibility to general medical and surgical hospitals by public transport was measured through a

13 two-step floating catchment area method, considering both service-to-population ratios and travel time to

14 these health services. For each metropolitan area, the average accessibility of all census tracts and the

15 average accessibility of the $20 \%$ most vulnerable census tracts were calculated. To quantify vertical equity,

16 an indicator was subsequently developed using the Spearman's rank correlation coefficient between

17 accessibility and vulnerability.

\section{Main findings:}

19 Within metropolitan areas (except for Vancouver), residents of vulnerable census tracts generally have 20 greater access to hospitals by public transport. Yet, larger metropolitan areas (Calgary, Toronto-Hamilton, 21 and Vancouver) tend to underperform in terms of vertical equity and average accessibility. In line with this,

22 vulnerable census tracts in these metropolitan areas are characterized by the lowest levels of accessibility

23 compared to vulnerable census tracts in the other metropolitan areas.

\section{Conclusion:}

25 This research highlights the challenges associated with the suburbanization of poverty in large Canadian 26 metropolitan regions and the need to provide efficient public transport services to reach hospitals located in 
1 the periphery. It also shed lights on the necessity to conduct more empirical research to inform the 2 implementation of guidelines in terms of accessibility to healthcare in Canada. This study is of relevance to 3 researchers, planners and policy-makers wishing to improve accessibility to healthcare, especially for 4 vulnerable populations. 


\section{1. INTRODUCTION}

2 The ability to access appropriate healthcare services has been at the centre of Canadian healthcare policy

3 for decades. One of the five pillars of The Canada Health Act states that "persons must have reasonable and 4 uniform access to insured health services, free of financial or other barriers. No one may be discriminated against on the basis of such factors as income, age, and health status." (Parliament of Canada, 1985) There

6 has, however, been extensive debate on how to interpret this notion of accessibility, both in government and academia (Wilson \& Rosenberg, 2004). In academia, the interpretation of accessibility has generally fallen into two distinct categories: potential and realized access to healthcare (Guagliardo, 2004; Wilson \& Rosenberg, 2004). Potential access is considered to be a function of the geographic distribution and supply of healthcare services, while realized access refers to actual utilization rates (Joseph \& Phillips, 1984).

11 Health Canada, on the other hand, specifies that accessibility can refer to both socio-economic access

12 (related to medical charges and a patient's age or health status) and the physical availability of "medically 13 necessary services" (Health Canada, 2017). Nevertheless, there seems to be a consensus that a form of 14 geographical or spatial access to healthcare falls within the concept of accessibility as defined in the Canada 15 Health Act, and can therefore be interpreted as a 'right'. Such debate on a 'right' to access healthcare 16 services is not unique to the Canadian context and is present in both developed and developing regions 17 around the world. Considering the existence of spatial factors that increase barriers to access health services, 18 inadvertently disadvantaging already vulnerable populations, it is especially pertinent to develop a 19 methodology to measure spatial accessibility to healthcare and examine it from an equity perspective. 20 Furthermore, the inclusion of multiple metropolitan regions allows identifying common trends and specific 21 challenges using a benchmarking approach.

Canada's metropolitan growth has largely followed a car-oriented development which has resulted

23 in the spatial dispersion of activities. This poses important accessibility challenges for populations that do 24 not have access to a private vehicle. As matter of fact, a significant proportion of low-income households 25 does not own a private vehicle and depend on public and active transport for their mobility needs, including 26 accessing healthcare services (Paez, Ruben, Faber, Morency, \& Roorda, 2009). Furthermore, in Canada's 
1 major cities, an aging population progressively relies on public transport to access healthcare facilities due

2 to conditions resulting in the loss of driving ability, which are more frequent at older ages (Chihuri et al.,

3 2016). At the same time, hospitals have recently begun to increase parking costs, thus making an ever-larger

4 share of population dependent on public transport.

5 This paper aims to quantify the spatial accessibility to healthcare services (specifically general

6 medical and surgical hospitals) by public transport across eight major Canadian metropolitan areas, and

7 compare accessibility to healthcare across vulnerable populations to generate a benchmarking level of access

8 that can be compared across different regions. Spatial accessibility to healthcare is measured using a two-

9 step floating catchment area method (2SFCA), incorporating both the spatial relationship between supply

10 (captured by the number of beds) and demand for services (population), and competition effects for scarce

11 resources (Mao \& Nekorchuk, 2013). The generation of accessibility measures for multiple cities allows

12 identifying some of the main challenges to be addressed to improve accessibility to healthcare in Canada,

13 especially for vulnerable populations, and sheds light on the factors to take into account in the allocation of

14 health and transport resources.

15 The rest of this paper is structured as follows. In section 2, relevant literature is presented, while in

16 section 3 the data and methodology used to calculate spatial accessibility are discussed. Section 4 presents

17 the results of the accessibility calculations, and provides a comparison between Canadian cities in terms of

18 average accessibility and the distribution of accessibility across socio-economic groups. Section 5 then

19 concludes this paper.

\section{2. LITERATURE REVIEW}

21 A vast body of literature has established links between access to health services and health outcomes.

22 Increased distances to healthcare facilities, for example, are associated with lower rates of utilization of the

23 healthcare system (Haynes, Lovett, \& Sunnenberg, 2003; Hiscock, Pearce, Blakely, \& Witten, 2008), often

24 exacerbating pre-existing inequities in health status (Guagliardo, Ronzio, Cheung, Chacko, \& Joseph, 2004;

25 Korda, Butler, Clements, \& Kunitz, 2007). However, despite the substantial importance of these links, two 
1 distinct areas of research have independently been preoccupied with measuring and understanding access

2 to healthcare services.

3 Transport scholars and practitioners usually measure access to (health) services through

4 accessibility, which is defined as the ease of reaching destinations (Boisjoly \& El-Geneidy, 2017; Delmelle

5 \& Casas, 2012; Geurs \& van Wee, 2004; Handy \& Niemeier, 1997). Accessibility, first defined as the

6 potential of opportunities for interaction (Hansen, 1959), is often operationalized through the number of

7 opportunities that can be reached from a certain point in space within a specified time limit, e.g. the number

8 of hospitals that can be reached within 30 minutes of travel time- what is known as cumulative accessibility

9 (Wickstrom, 1971). Recent research has also proposed the use of variable thresholds to measure the number

10 of healthcare clinics reachable in a region (Paez, Mercado, Farber, Morency, \& Roorda, 2010). Gravity-

11 based accessibility measures further expand on the cumulative metrics by discounting services by distance;

12 the further a service is, the less it contributes to accessibility (El-Geneidy \& Levinson, 2006; Weibull, 1976).

13 Health researchers often opt for simpler metrics of accessibility such as the distance to the nearest

14 service, service-to-population ratios, or service areas (Apparicio, Abdelmajid, Riva, \& Shearmur, 2008;

15 Neutens, 2015). The average travel time from a point in space to all health providers in a certain region has

16 also been used for this purpose (Guagliardo, 2004). In contrast with the transport-oriented metrics, these

17 measures have the advantage of being easier to calculate and communicate to a large variety of audiences.

18 The above-mentioned metrics are however incomplete and cannot fully capture all dimensions

19 related to spatial access to health services. Cumulative and gravity-based accessibility measures, for

20 example, do not take into account demand side considerations; they assume that services will be fully

21 available to residents, regardless of their capacity - one service $2 \mathrm{~km}$ away from one person would be equal

22 to one service at a distance of $2 \mathrm{~km}$ away from a million individuals (Luo \& Wang, 2003). The distance to

23 the nearest service and service area metrics exhibit the same issues. The service-to-population ratio, on the

24 other hand, does consider demand, but gives no indication as to how far individuals would need to travel to

25 reach a service and is often calculated via aggregated areal units that are too large to conclude meaningful

26 results (Guagliardo, 2004; Love \& Lindquist, 1995). 
In response to the shortcomings associated with these metrics, the two-step floating catchment area method (2SFCA) was developed by Joseph and Bantock (1982) (although not named as such) and later Luo and Wang (2003), the scholars responsible for coining the term. This method combines both demand and supply side metrics, and can accurately control for travel impedance, capacity restrictions and competition for services (Neutens, 2015). As the name suggests, the 2SFCA consists of two stages, where in the first step the service-to-population ratio is computed for each service, and then in the second step cumulative or gravity-based accessibility is calculated based on the service-to-population ratios (Mao \& Nekorchuk, 2013;

8 McGrail \& Humphreys, 2009; Tang, Chiu, Chiang, Su, \& Chan, 2017). In essence, the 2SFCA sums the 9 service-to-population ratios of services that can be reached from a specified point in space. As demonstrated 10 by Luo and Wang (2003), this is equivalent to the 'competitive' measures of access that are commonly used 11 in transport research to measure accessibility to job opportunities (Andersson, Haltiwanger, Kutzbach, 12 Pollakowski, \& Weinberg, 2014; El-Geneidy \& Levinson, 2011; Korsu \& Wenglenski, 2010; Shen, 1998). Two competing notions of equity exist in transport research: horizontal and vertical equity (Foth, 14 Manaugh, \& El-Geneidy, 2013; Lucas, van Wee, \& Maat, 2016; van Wee \& Geurs, 2011). A distribution is considered horizontally equitable if everyone has the same accessibility, regardless of his or her personal socio-economic characteristics. The allocation of resources is vertically equitable if those with the highest need also have access to the most resources, where need is usually measured through socio-economic status.

18 Accessibility metrics have often been employed to measure the equity of the spatial distribution of 19 opportunities (Golub \& Martens, 2014; Grengs, 2015; Guzman, Oviedo, \& Rivera, 2017). However, most 20 health studies measuring access to healthcare facilities have paid little attention to how both spatial and non21 spatial characteristics, such as socio-economic status, relate to access levels (Bissonnette, Wilson, Bell, \& 22 Shah, 2012). Furthermore, while many scholars have used metrics such as the Gini coefficient or the Lorenz 23 curve to measure horizontal equity (Lucas et al., 2016), little research has been undertaken to quantify 24 vertical equity. To our knowledge, only one study has undertaken this task, by calculating vertical equity 25 through a spearman correlation coefficient, although the authors did not apply this to an accessibility metric 26 (Mortazavi \& Akbarzadeh, 2017). This is, therefore, the first study to develop a vertical equity measure to 
1 assess the distribution of accessibility levels across socio-economic groups. This is also the first study, to

2 the authors' knowledge, to conduct a comparative assessment of accessibility to hospitals by public transport

3 across multiple metropolitan regions, thereby allowing to identify common trends. The study combines the

4 accessibility measures developed with the 2SCFA with household income data to provide a deeper

5 understanding of the socio-spatial patterns of accessibility to healthcare.

\section{$6 \quad 3$. CONTEXT}

7 An overview of the eight areas that were examined in this study can be seen in Figure 1. Their main

8 characteristics are presented in Table 1, from the largest metropolitan region to the smallest. These 8 cities

9 were selected based on the availability of data, their population size as well as their geographic diversity.

10 First, the 10 largest metropolitan regions in Canada were considered. Of these 10 metropolitan regions, only

11 those for which the data on the number of beds per hospital was available were selected. Three cities, among

12 the 10 largest in Canada, were excluded due to absence of the data on the number of beds in the province of

13 Quebec. As a result, 7 of the largest metropolitan regions were included in this study. These consists of all

14 the regions listed in Figure 1, with the exception of Halifax. The metropolitan region of Halifax, although

15 not among the 10 largest metropolitan regions, was included to have at least one region from the eastern

16 part of Canada.

17 


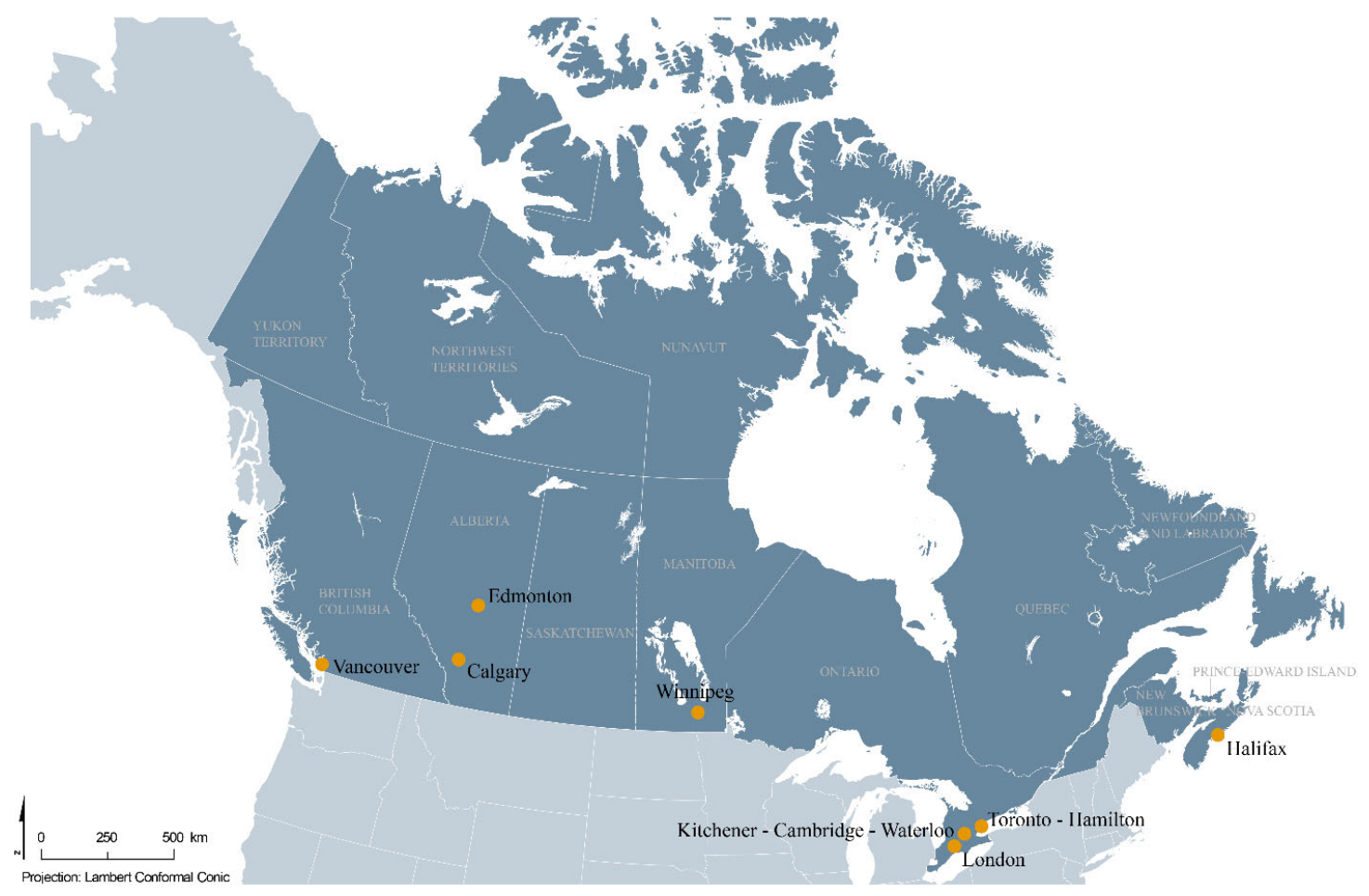

2 Figure 1 Overview of the 8 metropolitan areas included in the study

The 8 metropolitan regions are diverse in terms of population, land use organization as well as

4 transport networks. The population ranges from around 7 million inhabitants in Toronto - Hamilton to

5 around 400,000 inhabitants in Halifax. The population density also varies greatly, from 865 to 73

6 inhabitants $/ \mathrm{km}^{2}$. In terms of high-capacity public transport services, Toronto - Hamilton has the most

7 extensive public transport network, with metro, light rail transit (LRT) and commuter rail networks.

8 Vancouver, Calgary, Edmonton and Kitchener - Cambridge - Waterloo are all served by a LRT network,

9 while Edmonton has a Bus Rapid Transit. Finally, no high-capacity public transport system operates in

10 London and Halifax. Interestingly, average public transport commute times are not necessarily smaller in

11 smaller metropolitan regions. For example, Halifax and Toronto - Hamilton have similar public transport

12 commute times (60 minutes and 59 minutes respectively). The variations in average public transport

13 commute times are probably influenced by land use organization as well as the performance of the public

14 transport networks. Note that the hospital beds data is discussed in the next section. 
It is important to emphasize the high diversity of metropolitan regions that were considered in this

2 study and to recognize the presence of context-specific factors that inevitably influence the levels of

3 accessibility by public transport. In line with this, the purpose of this study is to identify the main challenges,

4 in terms of accessibility to healthcare by public transport, that are present in Canada's large metropolitan

5 regions. These challenges can be either common to several metropolitan regions or specific to one or a few

6 of them.

In terms of transport equity in Canadian metropolitan regions, low-income households typically

8 reside in more central areas that are better served by public transport. For example, in Toronto - Hamilton

9 and Montreal, low-income individuals have better average accessibility to jobs by public transport than their

10 counterpart (El-Geneidy et al., 2016; Foth et al., 2013). Yet, an increasing proportion of low-income

11 households individuals are pushed towards the suburbs, partly due to increasing housing costs (Ades,

12 Apparicio, \& Séguin, 2016; Pavlic \& Qian, 2014). With respect to travel behavior, low-income individuals

13 tend to travel shorter distances and rely more importantly on public transport (Statistics Canada, 2016). The

14 shorter travelled distances often result from a proximity strategy, given the lack of available options to travel

15 longer distances. For example, individuals who do not have convenient options to travel long distances (e.g.:

16 lack of access to a car or performant public transport services) may search for employment opportunities

17 only in their vicinity. However, this proximity strategy becomes problematic when looking at hospital

18 access. Hospitals are not typically not available at the local level, which requires an important share of the

19 population to travel longer distances to access theses services. It is therefore especially important to assess

20 accessibility to healthcare for population who rely on public transport.

21 Table 1 Information on population, public transport and hospital beds in the eight metropolitan regions

\begin{tabular}{|l|c|r|r|r|r|r|}
\hline $\begin{array}{c}\text { Metropolitan } \\
\text { region }\end{array}$ & Population & $\begin{array}{c}\text { Population } \\
\text { density } \\
\left(\mathbf{p o p} / \mathbf{k m}^{2}\right)\end{array}$ & $\begin{array}{c}\text { Average } \\
\text { public } \\
\text { transport } \\
\text { commute } \\
\text { time } \\
\text { (min) }\end{array}$ & $\begin{array}{c}\text { High-capacity } \\
\text { public } \\
\text { transport }\end{array}$ & $\begin{array}{c}\text { Number of } \\
\text { beds }\end{array}$ & $\begin{array}{c}\text { Population- } \\
\text { bed ratio } \\
\text { (number of } \\
\text { beds/1,000 } \\
\text { people) }\end{array}$ \\
\hline $\begin{array}{l}\text { Toronto- } \\
\text { Hamilton }\end{array}$ & $7,055,433$ & 862 & 59 & Commuter rail, & 14670 & 2.08 \\
\hline
\end{tabular}




\begin{tabular}{|c|c|c|c|c|c|c|}
\hline & & & & $\begin{array}{r}\text { Light rail } \\
\text { transit }\end{array}$ & & \\
\hline Vancouver & $2,463,431$ & 855 & 50 & $\begin{array}{r}\text { Light rail } \\
\text { transit }\end{array}$ & 6967 & 2.83 \\
\hline Calgary & $1,392,609$ & 273 & 58 & $\begin{array}{r}\text { Light rail } \\
\text { transit }\end{array}$ & 2616 & 1.88 \\
\hline Edmonton & $1,321,426$ & 140 & 64 & $\begin{array}{r}\text { Light rail } \\
\text { transit }\end{array}$ & 3301 & 2.50 \\
\hline Winnipeg & 778,489 & 147 & 43 & $\begin{array}{r}\text { Bus rapid } \\
\text { transit }\end{array}$ & 2623 & 3.37 \\
\hline $\begin{array}{l}\text { Kitchener- } \\
\text { Cambridge- } \\
\text { Waterloo }\end{array}$ & 523,894 & 480 & 46 & $\begin{array}{r}\text { Light rail } \\
\text { transit }\end{array}$ & 804 & 1.53 \\
\hline London & 494,069 & 186 & 49 & - & 1715 & 3.47 \\
\hline Halifax & 403,390 & 73 & 60 & - & 1199 & 2.97 \\
\hline
\end{tabular}

1 Sources: CIHI, 2018 hospital inventory (CIHI, 2018); Statistics Canada Census 2016 (Statistics Canada, 2 2016)

\section{MATERIAL AND METHODS}

Two distinct data sources were used to compute accessibility levels. Information on general healthcare services was obtained through the Canadian Institute for Health Information (CIHI). More specifically, the number of beds staffed and in operation for hospital services in Canadian provinces in 20152016 was used (CIHI, 2018). CIHI provides the total number of beds associated with each hospital, and the information was subsequently geocoded using a Google API using hospital names and addresses. It is important to note that these data were not available for the Province of Quebec. Accordingly, metropolitan regions in Quebec are excluded from the analysis. While former studies have measured accessibility to primary healthcare based on medical clinics or community pharmacies (Apparicio et al., 2008; Law, Dijkstra, Douillard, \& Morgan, 2011; Paez et al., 2010), our study specifically focuses on hospital services, which reflects access to emergency rooms, major outpatient clinics, and specialized care. This kind of healthcare service was selected for two reasons: the supply of this type of service is more consistent across provinces (no registration required to access most of these services, except specialized ones) and the geographic access to such services typically implies longer travel distances, which may require individuals to travel by car or by public transport. The number of beds is used in this study to better capture the supply at each hospital, as it reflects the size of the hospital and potentially the diversity of healthcare services available (assuming larger hospitals offer more services). Other detailed information such as number of 
1 doctors in an emergency care unit or at the hospital can also be used as proxies, yet such information was

2 not available across all regions. The total number of beds available in all hospitals in each of the eight

3 regions, as well as the bed-population ratio (number of beds per 1,000 inhabitants), are presented in Table

4 1. Public transport schedules across the eight metropolitan regions were downloaded in the General Transit

5 Feed Specification (GTFS) format from their respective transport agencies. All schedules were obtained for

6 May 2017 where available, or as close to this date as possible depending on the release date of the GTFS

7 data by the different agencies. If multiple agencies served a single metropolitan area, the schedules from all

8 agencies were obtained with overlapping schedule dates. Public transport schedules were digitized into a

9 geographic information system through the Add GTFS to a network dataset add-on for ArcGIS. To calculate

10 accessibility to healthcare services, a travel time matrix was generated for each region, providing the travel

11 time from all census tracts (CT) to every CT where at least one hospital is located. The travel times were

12 obtained by calculating the fastest route between CT centroids within each metropolitan area at $10 \mathrm{AM}$ on

13 a regular Tuesday. The fastest route algorithm incorporated walking time from the CT centroid (origin) to

14 the public transport station, waiting time, in-vehicle time (as determined by the transit schedule), any

15 transfer time, and walking time from the last stop to the CT centroid (destination). The $10 \mathrm{AM}$ leaving time

16 was chosen to account for an off-peak public transport service level.

17 A two-step floating catchment area method was subsequently used to calculate spatial accessibility.

18 First, a service-to-population ratio $V_{j}$ was calculated for each hospital, taking into account the total

19 population that can reach the service within 45 minutes by public transport:

$$
V_{j}=\frac{s_{j}}{\sum_{k} P_{k} f\left(t_{k j}\right)} \text { and } \mathrm{f}\left(t_{k j}\right)=\left\{\begin{array}{l}
1 \text { if } t_{k j} \leq 45 \text { minutes } \\
0 \text { if } t_{k j}>45 \text { minutes }
\end{array}\right.
$$

23 Where $V_{j}$ represents the number of beds per person, $j$ denotes a healthcare service, $S_{j}$ represents the capacity 24 of this service (number of beds), $P_{k}$ is the population in census tract $k$ and $t_{k j}$ is the travel time between 25 census tract $k$ and healthcare service $j . P_{k} f\left(t_{k j}\right)$ can therefore be interpreted as the population at location 
$1 \quad k$ that can reach the service within 45 minutes by transit. Second, accessibility for each census tract was

2 computed by summing the service-to-population ratios for the services that can be reached from each census

3 tract centroid within 45 minutes:

4

$$
A_{i}=\sum_{j} V_{j} \mathrm{f}\left(t_{j i}\right) \text { and } \mathrm{f}\left(t_{j i}\right)=\left\{\begin{array}{l}
1 \text { if } t_{j i} \leq 45 \text { minutes } \\
0 \text { if } t_{j i}>45 \text { minutes }
\end{array}\right.
$$

6

7 where $i$ denotes a census tract, $V_{j}$ is the service-to-population ratio for service $j$, and $t_{j i}$ is the travel time

8 between $j$ and $i$ via public transport. The accessibility measure thus counts the number of beds available

9 within 45 minutes, and adds the service-to-population ratio for each service. For simplicity reasons, 10 accessibility is expressed as the number of beds per 1,000 people.

11 Regarding the selection of a travel time threshold, this study adopts a normative approach (i.e.:

12 prescriptive) (Paez, Scott, \& Morency, 2012). Normative accessibility is typically defined following an

13 expectation from the policy-makers, based on "how far ought to or how far it is reasonable for people to

14 travel". This is in line with the accessibility principle of the Canada Health Act, stating that all individuals

15 must have reasonable access to health services. As there is no guidelines as to what is considered a

16 reasonable access in terms of travel time and distance, a decision had to be taken to establish a threshold in

17 this study. Given that this reasonable access is required by the federal government and the lack of context-

18 specific guidelines, it is adequate to adopt a constant threshold across metropolitan regions. Indeed, it is

19 possible to argue that all Canadians should be provided with similar level of access, whether they live in

20 Vancouver or Saskatoon.

21 As specialized healthcare is typically provided at the metropolitan level, rather than the 22 neighborhood level, the threshold was selected to reflect metropolitan accessibility. A 45-minute threshold

23 was selected, as is commonly used in transport planning to measure regional accessibility (Boisjoly \& El-

24 Geneidy, 2017). While it would have been relevant to adopt a threshold that is specific to healthcare 25 accessibility, no study, to the authors' knowledge, has been conducted to inform travel time behavior and 
1 thresholds in terms of accessibility to hospitals in Canadian metropolitans. The limitations and implications

2 related to the selection of a travel time threshold a further discussed in the Discussion and conclusion 3 section.

To assess the socio-spatial distribution of accessibility levels, a vulnerability index was computed representing the vulnerability of a census tract based on the characteristics of its population. From a transport perspective, equity suggests that those with the greatest needs should be prioritized (Pereira, Schwanen, \& Banister, 2017). As this study focuses on accessibility by public transport, vulnerability refers here to the 8 characteristics that increase the likelihood of an individual to rely on public transport. The literature 9 indicates that low-income individuals, recent immigrants, unemployed individuals, youth and elderly as 10 well as women are more likely to have limited access to a private vehicle and accordingly depend on public 11 transport. In the context of the study, the most relevant variables, available at the census tract level, were 12 identified based on study conducted in Canada by Foth et al. (2013): (i) median household income (I), (ii) 13 unemployment rate (U), (iii) the percentage of the population that has immigrated within the last 5 years 14 (IM), and (iv) the percentage of households that spend more than $30 \%$ of their total income on housing rent 15 (R). All variables were obtained from the 2016 Census of Canada and were then standardized through zscores. The final vulnerability index of a census tract is given by the following formula, where $Z_{X}$ represents

17 the z-score of variable $X$ :

$$
\text { Vulnerability }=-Z_{I}+Z_{U}+Z_{I M}+Z_{R}
$$

Vertical equity was then calculated for each metropolitan region to assess the distribution of 20 accessibility to health services (number of beds in a hospital that are reachable within 45 minutes of travel 21 time divided by the population that can reach the service within 45 minutes) based on the vulnerability 22 indicator. The correlation between the vulnerability index and accessibility levels was generated through a 23 Spearman's rank correlation index. This approach builds on a previous research (Mortazavi \& Akbarzadeh, 24 2017) that use the Spearman's rank correlation index to quantify the vertical equity of the distribution of 25 public transport services. This approach assigns an accessibility rank and a vulnerability rank to each census 26 tract and assesses whether census tracts ranking high in terms of accessibility also rank high in terms of 
1 vulnerability index. A coefficient of 1 would mean that the accessibility ranking is exactly the same as the

2 vulnerability ranking, where the census tract with the highest accessibility also has the highest vulnerability

3 index, and the census tract with the lowest accessibility has the lowest vulnerability index. Accordingly, the

4 Spearman rank correlation coefficient indicates whether census tracts with high vulnerability (and

5 accordingly high potential needs in terms of transport and health) also have the highest accessibility, which

$6 \quad$ is desirable from a vertical equity perspective.

7 5. RESULTS

8 4.1. Accessibility and equity across metropolitan regions

9 Table 2 presents the mean census tracts accessibility (hereafter referred to as average accessibility) as well

10 as the minimum, the maximum and the standard deviation for all census tracts and for the $20 \%$ most

11 vulnerable census tracts. For ease of interpretation, the results are presented from the largest to the smallest

12 metropolitan region. Before going further into the analysis, it is important to note that the minimum

13 accessibility for all metropolitan regions is null, indicating that there is at least one census tract without

14 accessibility to hospitals by public transport in every region. Also, this study focuses on the mean

15 accessibility to compare the overall level of accessibility to hospitals provided in every metropolitan region,

16 but further studies could look into the variations within every metropolitan region. For example, Halifax

17 and London, the two smallest metropolitan regions in terms of population, exhibit large standard deviations

18 both for all census tracts and the $20 \%$ most vulnerable census tracts. They are also characterized by high

19 maximum values of accessibility, suggesting important differences between census tracts. Vancouver also

20 exhibits a large standard deviation for all census tracts, which is explained by the notably high accessibility

21 of one census tract. This is further discussed in the discussion section.

22 Table 2 Accessibility across metropolitan regions (number of beds/1,000 people)

\begin{tabular}{|l|r|r|r|r|r|r|r|r|}
\hline \multirow{2}{*}{$\begin{array}{l}\text { Metropolitan } \\
\text { region }\end{array}$} & \multicolumn{4}{|c|}{ All census tracts } & \multicolumn{3}{|c|}{$\mathbf{2 0 \%}$ most vulnerable census tracts } \\
\cline { 2 - 9 } & Mean & St. dev. & Min. & Max & Mean & St. dev. & Min. & Max \\
\hline $\begin{array}{l}\text { Toronto- } \\
\text { Hamilton }\end{array}$ & 2.24 & 2.66 & 0.00 & 16.68 & 2.80 & 2.33 & 0.00 & 9.14 \\
\hline
\end{tabular}




\begin{tabular}{|l|r|r|r|r|r|r|r|r|}
\hline Vancouver & 1.93 & 4.85 & 0.00 & 99.97 & 1.88 & 1.40 & 0.00 & 6.55 \\
\hline Calgary & 1.74 & 2.90 & 0.00 & 10.39 & 2.46 & 3.68 & 0.00 & 10.39 \\
\hline Edmonton & 2.61 & 3.30 & 0.00 & 19.45 & 4.48 & 3.34 & 0.00 & 9.93 \\
\hline Winnipeg & 3.43 & 3.43 & 0.00 & 22.57 & 4.50 & 3.36 & 0.00 & 13.19 \\
\hline $\begin{array}{l}\text { Kitchener- } \\
\text { Cambridge- } \\
\text { Waterloo }\end{array}$ & 1.66 & 1.61 & 0.00 & 3.76 & 2.98 & 1.32 & 0.00 & 3.76 \\
\hline $\begin{array}{l}\text { London } \\
\text { Halifax }\end{array}$ & 4.20 & 4.29 & 0.00 & 10.02 & 4.77 & 4.13 & 0.00 & 10.02 \\
\hline
\end{tabular}

1

2 A comparison between average accessibility to healthcare for all residents in a metropolitan area and the

average accessibility experienced by the residents in the $20 \%$ most vulnerable census tracts is shown in

Figure 2, again from the largest to the smallest metropolitan region. Looking first at the average accessibility to healthcare for all residents, the results show that the largest metropolitan regions (Toronto - Hamilton,

Vancouver and Calgary) exhibit the lowest accessibility values. Conversely, Halifax and London, the smallest metropolitan regions, have significantly higher levels of accessibility.

It is important to note that Halifax, London and Winnipeg, which have the highest average accessibility to healthcare for all residents, also have the largest number of beds relative to the population (respectively $2.97,3.47$ and 3.37 beds per 1,000 inhabitants - see Table 1). In contrast, KitchenerCambridge-Waterloo and Calgary have the lowest bed to population ratios (1.53 and 1.88 respectively), which results in low levels of accessibility. Interestingly, Vancouver exhibits a high ratio (2.83), similar to Halifax, but is characterized by a low average accessibility to healthcare. This suggests that, while the quantity of supply is an important determinant of accessibility, other factors come into play when examining accessibility to healthcare services, namely the spatial distribution of hospitals and the performance of the public transport network, two themes explored in the next section.

In terms of equity, vulnerable census tracts are characterized by higher accessibility to health services than the average of the region. In Halifax, this difference is most profound: residents in vulnerable 
census tracts can access $88 \%$ more services than average (accessibility values of 6.87 and 3.64 ,

2 respectively). Those residents also experience the highest accessibility value across all regions, although the

3 highest average accessibility (including all census tracts) is found in London. In contrast, Vancouver has

4 the lowest accessibility levels of all metropolitan areas with respect to the $20 \%$ most vulnerable census

5 tracts, almost 4 times less than the average for the $20 \%$ vulnerable census tracts in Halifax (accessibility

6 values of 1.74 and 6.87, respectively). Most notably, in Vancouver, vulnerable census tracts exhibit a lower

7 accessibility to healthcare services compared to the average of the metropolitan region. This reflects an

8 inequitable distribution of accessibility from a vertical equity standpoint, as vulnerable populations, which

9 are more likely to depend on public transport to access healthcare services, experience lower accessibility

10 than average.

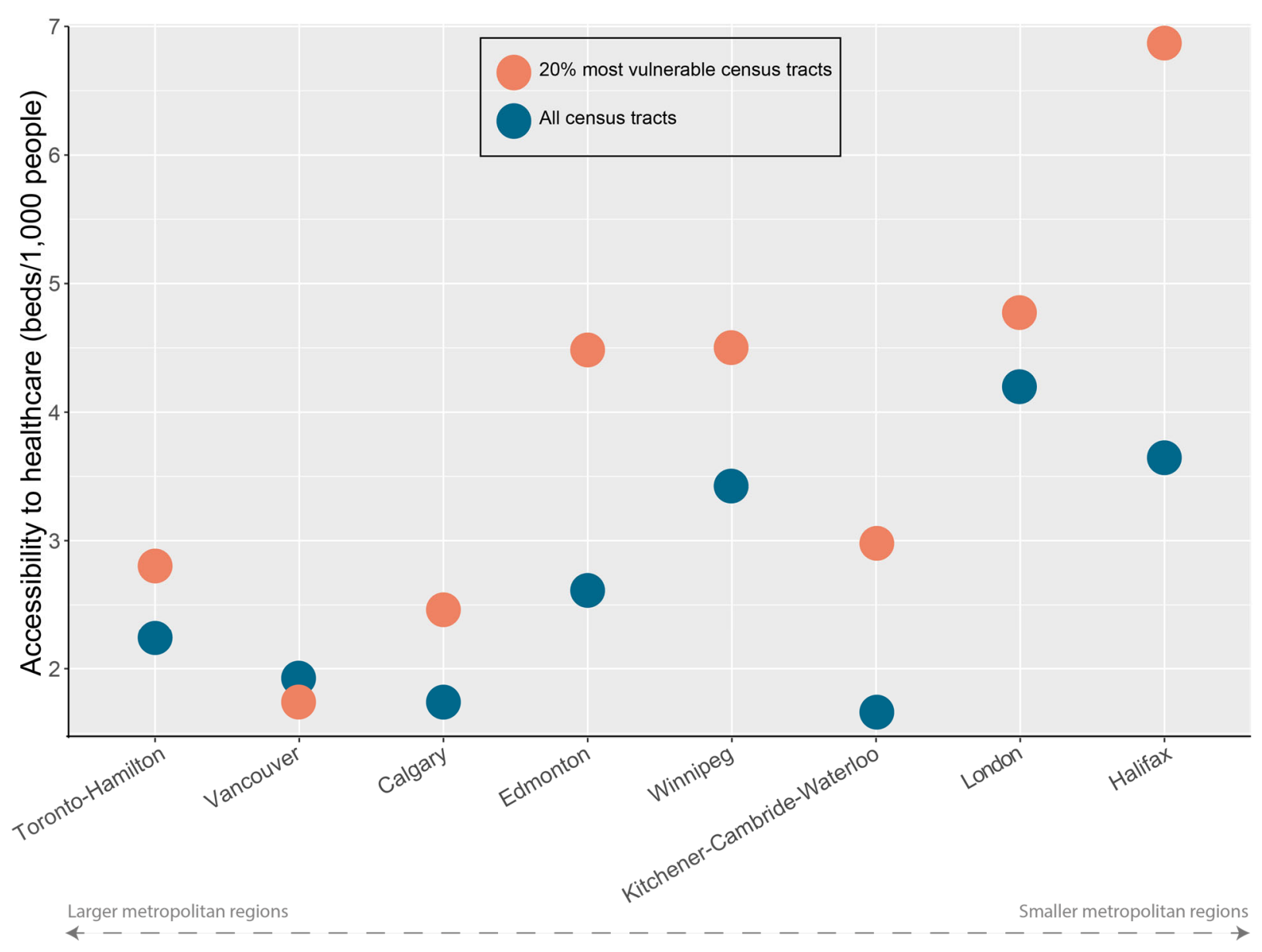

12 Figure 2 Comparison of accessibility levels across the eight metropolitan areas 
To further explore the socio-spatial distribution of accessibility across metropolitan regions, a

2 vertical equity indicator was calculated for each metropolitan region, using the Spearman's rank correlation

3 index. Figure 3 compares the eight metropolitan areas based on average accessibility to healthcare (x-axis)

4 and vertical equity of accessibility to healthcare (y-axis). The size of the circles corresponds to the size of

5 the metropolitan population. A city at the top right corner would represent the ideal situation: a region with

6 high levels of access to hospital beds overall, and where this access is equitably distributed across different

7 socio-economic groups, i.e., where the individuals residing in vulnerable census tracts typically experience

8 higher accessibility. Not surprisingly, Vancouver is amongst the metropolitan regions with the lowest

9 vertical equity indicator, given that the accessibility of vulnerable census tracts in Vancouver is lower than

10 the average of the region. Conversely, Halifax is characterized by a high vertical equity indicator as

11 vulnerable census tracts have a much higher accessibility than the average of the region. More generally,

12 metropolitan regions where the vulnerable census tracts exhibit the highest levels of accessibility across

13 Canada (Edmonton, Winnipeg, Halifax and London) perform well on both indicators. Conversely, in regions

14 with low average accessibility and low vertical equity (Calgary, Toronto-Hamilton and Vancouver),

15 vulnerable census tracts exhibit the lowest levels of accessibility to healthcare across Canada. This

16 highlights the difficulty to serve vulnerable census tracts when the average accessibility to healthcare is

17 already low.

18 Also, as a general trend, it appears that larger metropolitan regions tend to perform worse in terms

19 of vertical equity, with Calgary, Toronto-Hamilton, and Vancouver having the lowest vertical equity

20 indicators. One possible reason explaining this is the suburbanization of poverty, combined with the

21 concentration of healthcare services in the center. In contrast, smaller cities such as Halifax, London and

22 Kitchener-Cambridge-Waterloo and Winnipeg, are characterized by a high level of vertical equity. This is

23 likely explained by the fact that most of the vulnerable census tracts in these cities are located in or near the

24 downtown area, where several hospitals are located. Vulnerable census tracts in these cities therefore have

25 a high accessibility to healthcare relative to the rest of the region, resulting in a high vertical equity indicator. 
2 the exception of Kitchener-Cambridge-Waterloo, potentially explained by the low bed-population ratio)

3 both in terms of vertical equity and average accessibility to healthcare services. Conversely, larger

4 metropolitan areas exhibit low vertical equity and low average accessibility, which results in low

5 accessibility in the vulnerable census tracts. This is potentially due to low bed-population ratio (in Calgary

6 and Toronto-Hamilton) but also to the difficulty to serve a population that is spatially dispersed.

7 Accordingly, the results suggest that more efforts are needed in larger metropolitan regions to increase

8 accessibility to healthcare, especially for vulnerable census tracts.

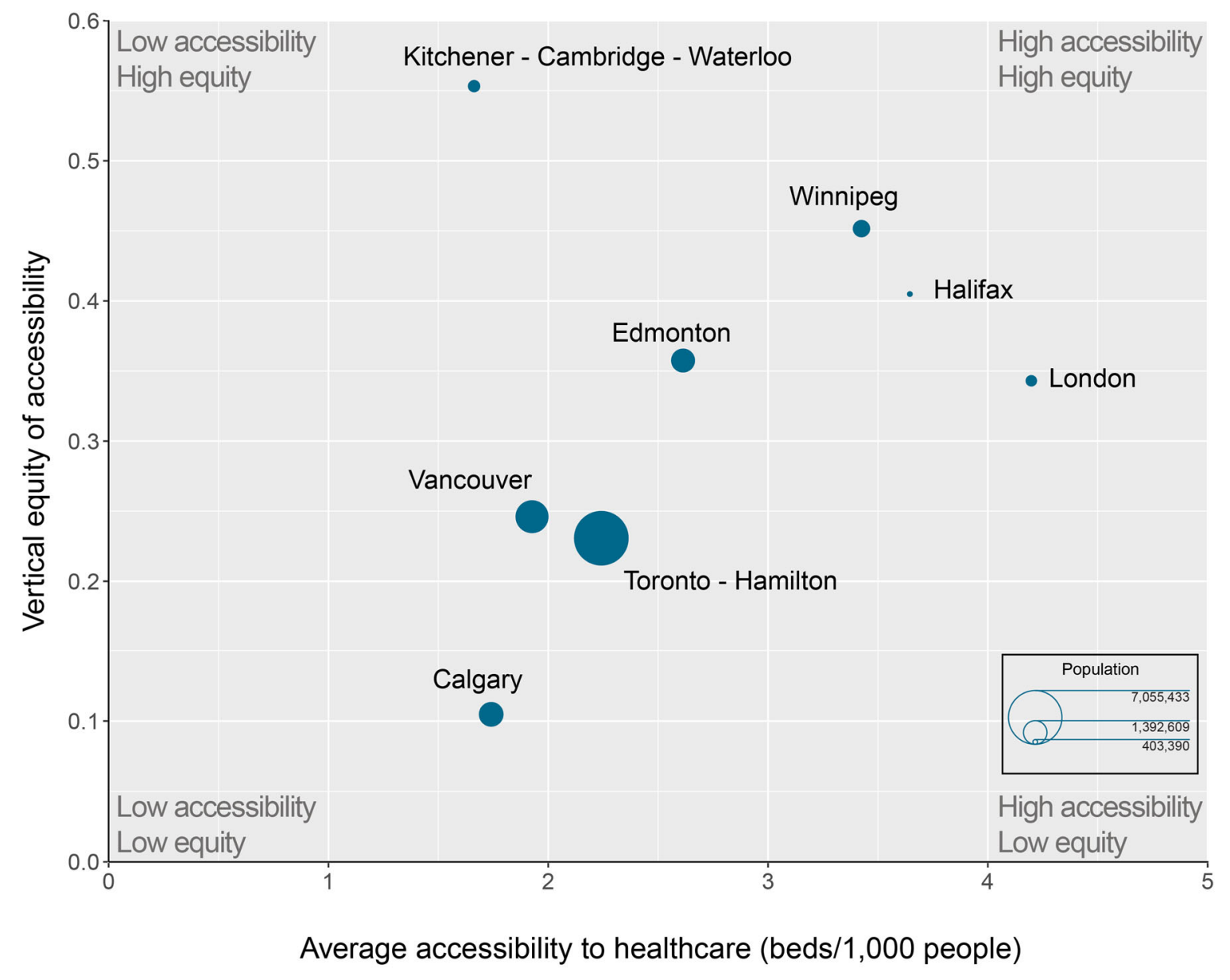

10 Figure 3 Accessibility and vertical equity across the eight metropolitan areas 
2 To better understand how metropolitan regions can support a greater and more equitable access to healthcare

3 services, this section explores the key land use and transport factors that lead to low accessibility to

4 healthcare in some of the regions, especially for vulnerable populations, as observed earlier in the results.

5 Figure 4 maps the accessibility to healthcare in Vancouver $(2,463,431$ inhabitants) and Winnipeg $(778,489$

6 inhabitations), with the $20 \%$ most vulnerable census tracts identified with a black outline. These two

7 metropolitan regions were selected as they demonstrate the contrasts between large and small metropolitan

8 regions, and highlight several of the issues that metropolitan regions are facing in terms of accessibility to

9 healthcare.

With respect to the average accessibility to healthcare, we see that, in both cases, the fringes of the metropolitan region experience low levels of accessibility, given the lack of hospitals and of public transport

12 services. Most notably, we see that important discrepancies in accessibility exist between the center and the

13 periphery in Winnipeg: whereas several central census tracts have an accessibility level above 6 , all census

14 tract in the periphery are characterized by a null accessibility, largely due to the limited public transport

15 service in peripheral areas. The detailed assessment of accessibility reveals an uneven distribution across

16 the region, although the average accessibility of the region is high. This is also the case in smaller

17 metropolitan regions such as London and Halifax. It is important to note that, while most vulnerable census

18 tracts are located in central areas in these regions, resulting in a high vertical equity indicator, there are

19 certainly vulnerable populations residing in the periphery. Smaller metropolitan regions should thus

20 concentrate on these populations and explore interventions that could support a high level of accessibility

21 by public transport to hospitals for these population groups.

22 Turning now to the Vancouver metropolitan region, where average accessibility is low, we see that

23 very few census tracts have a high level of accessibility (above 6). Most hospitals that are located in the

24 periphery of the region are only accessible to a limited number of census tracts in their surroundings, which

25 limits the level of accessibility by public transport experienced by residents outside the central areas and not

26 in the immediate surroundings of these hospitals. Conversely, this explains the high accessibility of the few 
census tracts that have access to these suburban hospitals. Improving public transport services to these

2 hospitals could lead to an increased accessibility in the periphery. In other words, the decentralization of

3 services in Vancouver should be accompanied by improved public transport services if one wishes to support

$4 \quad$ high levels of accessibility by public transport to healthcare in the suburban areas.

In terms of vertical equity, we see that most of the vulnerable census tracts are located away from

6 the city center, where there is a high concentration of hospitals. As a result, vulnerable census tracts

7 experience lower levels of accessibility. In order to support high accessibility in vulnerable census tracts

8 that are not located in the center, it is essential to support the decentralization of healthcare services,

9 considering where vulnerable census tracts are located, and to improve the provision of public transport

10 services to these hospitals. For example, there is a cluster of vulnerable census tracts in the south-east of the

11 region with an accessibility between 0 and 2 . Accessibility from these census tracts could be improved by

12 providing more services in that region (opening new hospitals or adding more beds within the existing ones),

13 or by improving accessibility by public transport to the surrounding hospitals, especially those that are

14 currently characterized by a low competition (to the South for example). This would also contribute to

15 improving the level of accessibility by public transport to healthcare services in the region.
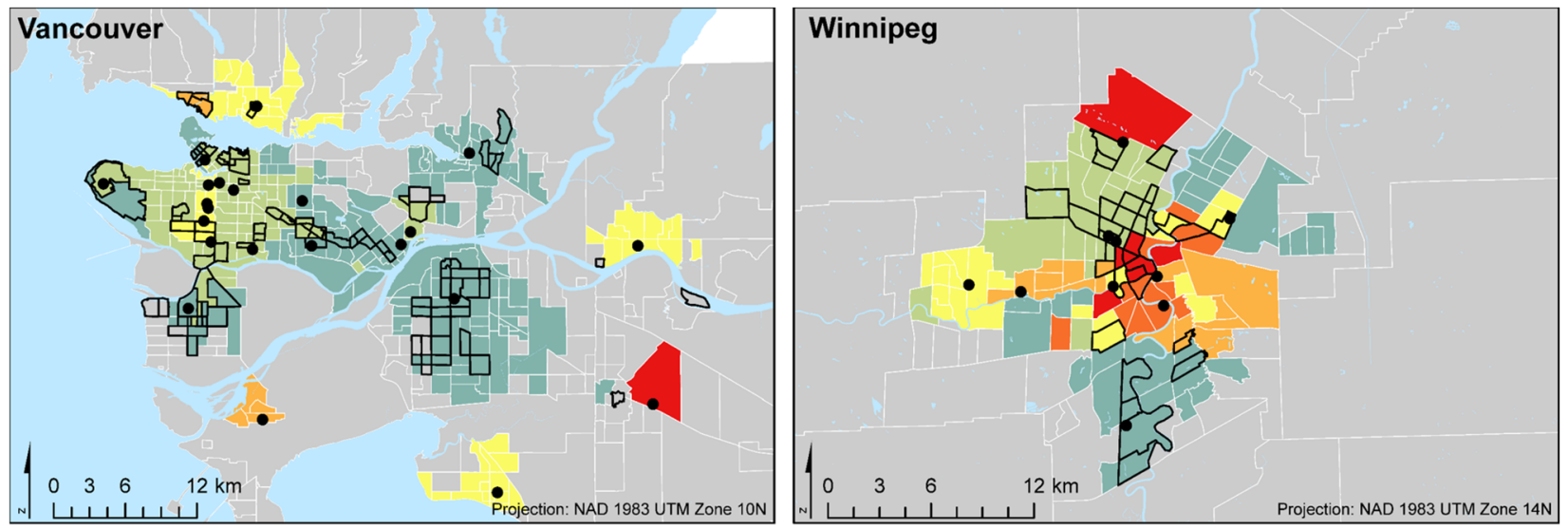

- Hospital

$20 \%$ most vulnerable census tracts

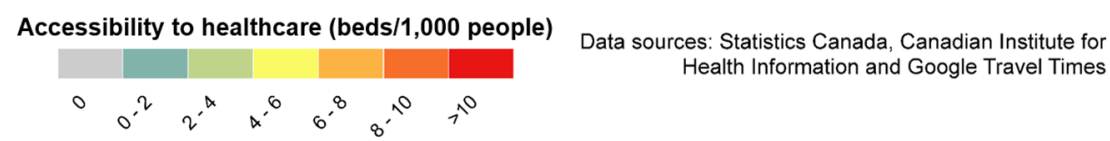

Figure 4 Accessibility to healthcare in Vancouver and Winnipeg, Canada 
It is important to note that similar patterns, although not as pronounced, are present in Calgary and

2 in Toronto-Hamilton. More generally, research conducted in large Canadian metropolitan regions has

3 shown that a growing proportion of low-income households locate in the inner suburbs of these regions

4 (Ades et al., 2016; Pavlic \& Qian, 2014). The suburbanization of poverty, in addition to the lack of efficient

5 public transport services in the suburbs, represents a significant challenge in providing adequate access to

6 healthcare services in Canada. The suburbanization of poverty is not unique to Canadian cities, but is present

7 in medium- and high-income countries around the world (Cooke \& Denton, 2015; Hochstenbach \& Musterd,

8 2017; Kneebone, 2017; Raphael \& Stoll, 2010). The concentration of low-income households in the suburbs

9 and urban fringes is also a significant problem in several cities of the Global South, namely in Latin America,

10 where such spatial segregation has been around for many decades (Blanco \& Apaolaza, 2018; Keeling,

11 2008). This study demonstrates that the development of land use and public transport interventions in

12 suburban areas would substantially contribute to higher levels of accessibility to healthcare overall, and

13 more importantly for vulnerable populations. Furthermore, the methods applied in this paper can be applied

14 in other areas around the world facing similar issues to help setting priority interventions to increase 15 accessibility to healthcare services.

\section{6. DISCUSSION AND CONCLUSION}

17 This study has examined spatial accessibility to general medical and surgical hospitals across eight 18 metropolitan regions in Canada accounting for both the travel times by public transport and the service-to-

19 population ratio, where the number of beds is used as proxy for the level of service. The study uses a 20 benchmarking approach to compare access to healthcare services within 45 minutes of travel time. Results

21 find that in all cities except for Vancouver, the socio-spatial distribution of accessibility to health services

22 is vertically equitable: residents in the $20 \%$ most vulnerable neighbourhoods live in areas with higher

23 accessibility than average for their cities. The groups with the highest needs thus tend to also experience the

24 highest accessibility. This advantage is, however, less pronounced (or simply absent) in larger metropolitan

25 regions (Calgary, Toronto-Hamilton and Vancouver), which also exhibit relatively low average accessibility 
1 overall. As a result, vulnerable census tracts in these large metropolitan regions exhibit the lowest levels of

2 accessibility to healthcare services by public transport across Canada. This is largely explained by the lack

3 of accessibility by public transport to the hospitals located in the peripheries, and the high proportion of

$4 \quad$ vulnerable households in the inner suburbs of the regions, resulting from the suburbanization of poverty that

5 many large cities around the world have been experiencing.

6 Improving accessibility by public transport to healthcare services in the suburbs could potentially

7 contribute to improving the well-being of individuals, especially for vulnerable groups. As a matter a fact,

8 a recent study found that the implementation of a high-speed rail in rural areas in South Korea increased

9 healthcare service utilization among cancer patients (Choi, Kim, \& Park, 2019). Although conducted in a

10 different context, the study suggests that improvements in public transport services can have beneficial

11 impacts on consultation rates. While studies conducted in the US and Canada demonstrated that better

12 accessibility by public transport to healthcare services is positively associated with healthcare utilization

13 (Cui et al., 2020; Syed, Gerber, \& Sharp, 2013), little is known on the impacts of public transport

14 interventions on changes in healthcare utilization. As indicated by Syed et al. (2013) who conducted an

15 extensive review of the literature on the transport barriers to accessing healthcare services, this is an

16 important research gap.

17 The study has demonstrated how different indicators can be used to assess access to healthcare in

18 different regions. In metropolitan regions with low levels of accessibility and also low vertical equity,

19 vulnerable census tracts typically experience lower levels of accessibility to healthcare. It is, however,

20 necessary to go beyond these indicators, as demonstrated in the previous section, to better understand the

21 socio-spatial distribution of accessibility to healthcare services and to provide context-specific

22 recommendations. For example, in Vancouver, several peripheral hospitals have low competition and

23 improving public transport to these hospitals would significantly help in increasing vertical equity and

24 average accessibility. In the case of Winnipeg, the specific analysis of the metropolitan region depicts high

25 regional inequities between the centre and the periphery, although the region as a whole has a high average 
accessibility. Improving public transport to reach hospitals from the periphery would contribute to a more even spatial distribution.

Overall, while similar trends can be observed in different metropolitan regions, context-specific interventions are required to improve access to healthcare. In line with this, more efforts are required to assess how access to healthcare is considered in public transport planning and health policies in the different regions. Although the linkages between health and transport are increasingly considered by policy-makers and planners (Schoner et al., 2018), the focus lies mainly within active transport, physical activity and

8 exposure to noise and pollution (Cohen, Boniface, \& Watkins, 2014; Mueller et al., 2017). Since public

9 transport schedule data were made publicly available at the beginning of the 2000 s, several measures of 10 accessibility by public transport to healthcare services have been developed by researchers (Apparicio et al., 11 2008; Higgs, Zahnow, Corcoran, Langford, \& Fry, 2017; Mao \& Nekorchuk, 2013; Martin, Jordan, \&

12 Roderick, 2008; Neutens, 2015). Yet, little is known on how these measures are incorporated into planning 13 practices, and more efforts are needed to identify the barriers and opportunities to jointly address public 14 transport and healthcare access planning. Furthermore, whereas this study discussed how various land use and transport characteristics yield different accessibility patterns, further studies could attempt to shed light on the determinants of accessibility through regression modelling.

A sensitivity analysis was conducted to assess the influence of the time threshold on the results. The same analysis was conducted with a 60-minute threshold and a 30 -minute threshold. There is no major

19 difference between the 45-minute and 60-minute analysis. Except for the city of Halifax, where accessibility

20 is noticeably lower with the 60-minute thresholds, the accessibility levels of each metropolitan region are

21 comparable. Furthermore, the differences in accessibility between the $20 \%$ most vulnerable census tracts

22 and the whole region are comparable, which leads to similar trends in vertical equity: the largest 23 metropolitan region (Calgary, Vancouver and the Greater Toronto and Hamilton region) exhibit the lowest 24 vertical equity, while Kitchener - Cambridge - Waterloo and Winnipeg exhibit higher levels of equity. The 25 30-minute threshold also yields similar trends in terms of vertical equity, Kitchener - Cambridge - Waterloo 26 and Winnipeg being among the most equitable regions, and the largest metropolitan regions exhibiting lower 
1 levels of accessibility. However, some differences in the ranking of the metropolitan regions in terms of

2 average accessibility are observable. It is important to note that variations in accessibility across

3 metropolitan regions are lower when using a 30-minute threshold, which might not fully capture the

4 differences between the regions. Given the consistency of the results between the 45-minute and 60-minute

5 thresholds, and given that the 45-minute threshold used in this study corresponds to the average commute

6 time (44.8 minutes) for public transport users in Canadian metropolitan areas (Statistics Canada, 2016), we

7 are confident that the main findings discussed is this study represent a meaningful comparison of

8 accessibility to healthcare across Canada's largest metropolitan regions. Furthermore, a recent study

9 conducted in these 8 metropolitan regions found a positive relationship between accessibility to hospitals

10 by public transport (with a 45-minute threshold) and the likelihood of healthcare utilization, thereby

11 confirming the policy relevance of such measure for the current study (Cui et al., 2020). Further studies

12 could build on the methodology presented in this study to assess the relevance of different thresholds in

13 terms of realized access to healthcare. In other words, at which point does the travel time, by public transport,

14 represents a barrier to access healthcare? It would also be relevant to conduct such studies in different cities

15 to assess whether different context-specific factors lead to different outcomes. For example, do individuals

16 in different metropolitan regions react differently to the same levels of accessibility? This would contribute

17 to informing the debate on the selection of travel time thresholds for accessibility to healthcare in Canadian

18 metropolitan regions.

\section{Study Limitations}

It is important to acknowledge the limitations of this study. First, accessibility to hospitals was

21 measured at the census tract level, using the centroid of the census tracts as the point of reference for

22 calculating travel times. This does not reflect the exact location of healthcare services, especially when

23 considering large census tracts (mostly located in the periphery of the region), and, as a result, travel times

24 to the hospitals might be under- or over-estimated. In most cases, the impact on the calculated accessibility

25 is minimal, as there is no or little difference in the travel time. In a few cases where the hospital is situated

26 at the boundary of a large census tract, a more important difference can be found between the travel time 
1 calculated using the centroids of the census tract and the travel time that would result from using the exact

2 location of the hospital. As large census tracts are typically located in the periphery where public transport

3 services are limited, the impacts (overestimation or underestimation) on accessibility, if any, are limited to

4 the few census tracts surrounding the hospital, the others being more than 45 minutes away no matter how

5 travel time is calculated. The results presented in this study are nonetheless representative of the general

6 patterns of accessibility at the metropolitan level. Further studies could be conducted using a finer spatial

7 resolution, to get a more detailed accessibility assessment for the peripheral areas. A second limitation is

8 that we used travel time at 10 AM to account for off-peak public transport service level, although individuals

9 may need to visit the hospital at any time of the day. In major public transport agencies, off-peak services

10 are generally slower due to the reduced number of vehicles operating and increase in waiting and transfer

11 times, while in-vehicle time is generally lower when compared to peak services. We expect that accessibility

12 to healthcare services during the peak periods will be higher due to increase in the levels of services, yet we

13 do not expect major variations in the spatial distribution of accessibility. Another important point is that we

14 did not consider travel time reliability in our study. While GTFS data represents public transport schedules,

15 which typically account for congestion, we are well aware that traffic conditions change from one day to

16 another. It would thereby be relevant to develop measures that account for such variations in the future. It

17 is also important to point out that while this study focused on accessibility to general medical and surgical

18 hospitals - which represents a key component of the universal healthcare system in Canada - further studies

19 could look more specifically at primary care. Yet, since the primary care systems function differently from

20 one province to another, province-specific analyses should be conducted to take into account these

21 differences. Further studies could also build on the present study to evaluate the impacts of differential levels

22 of accessibility to specialized care on vulnerable individuals. This would contribute to a better understanding

23 of what the accessibility metric presented in this study reflect in terms of actual healthcare services received.

24 In our study we used 45 minutes threshold to derive accessibility measures, future studies can use different

25 thresholds or apply other measures of accessibility such as gravity based measures if the data needed to 
generate such measures are available for all studied regions from an origin destination survey that includes health care services to recommend a decay function or a different travel time threshold.

Overall, this study provides a comprehensive view of accessibility to general medical and surgical

hospitals across eight Canadian cities and demonstrates the growing challenges that Canadian metropolitan regions, and potentially many other cities around the world, are facing in terms of equity and accessibility to healthcare services. Urban policy-makers and public health professionals could build on this study to assess the levels of access to healthcare across various socio-economic groups in their cities, and to subsequently implement policies aimed at improving overall accessibility and accessibility for vulnerable populations to healthcare services by public transport.

\section{REFERENCES}

Ades, J., Apparicio, P., \& Séguin, A. (2016). Is poverty concentration expanding to the suburbs? Analyzing the intra-metropolitan poverty distribution and its change in Montreal, Toronto and Vancouver. Canadian Journal of Regional Science/Revue canadienne des sciences régionales, 39(1/3), 23-37.

Andersson, F., Haltiwanger, J., Kutzbach, M., Pollakowski, H., \& Weinberg, D. (2014). Job displacement and the duration of joblessness: The role of spatial mismatch. National Bureau of Economic Research. Cambridge, MA.

Apparicio, P., Abdelmajid, M., Riva, M., \& Shearmur, R. (2008). Comparing alternative approaches to measuring the geographical accessibility of urban health services: Distance types and aggregationerror issues. International Journal of Health Geographics, 7(7).

Bissonnette, L., Wilson, K., Bell, S., \& Shah, T. I. (2012). Neighbourhoods and potential access to health care: The role of spatial and aspatial factors. Health \& place, 18, 841 - 853.

Blanco, J., \& Apaolaza, R. (2018). Socio-territorial inequality and differential mobility. Three key issues in the Buenos Aires Metropolitan Region. Journal of Transport Geography, 67, 76-84.

Boisjoly, G., \& El-Geneidy, A. (2017). How to get there? A critical assessment of accessibility objectives and indicators in metropolitan transportation plans. Transport Policy, 55, 38-50.

Chihuri, S., Mielenz, T., DiMaggio, C., Betz, M., DiGuiseppi, C., Jones, V., \& Li, G. (2016). Driving cessation and health outcomes in older adults. Journal of the American Geriatrics Society, 64(2), 332-341.

Choi, J., Kim, S., \& Park, M. (2019). Effects of high-speed rail on health-care service utilization. Journal of Transport \& Health, 12, 13-20. doi:https://doi.org/10.1016/i.jth.2018.11.004

ClHI. (2018). Hospital beds staffed and in operation, 2016-2017.

Cohen, J., Boniface, S., \& Watkins, S. (2014). Health implications of transport planning, development and operations. Journal of Transport \& Health, 1(1), 63-72. doi:https://doi.org/10.1016/j.jth.2013.12.004

Cooke, T., \& Denton, C. (2015). The suburbanization of poverty? An alternative perspective. Urban Geography, 36(2), 300-313.

Cui, B., Boisjoly, G., Wasfi, R., Orpana, H., Manaugh, K., Buliung, R., ... El-Geneidy, A. (2020). Spatial access by public transport and likelihood of healthcare consultations at hospitals Paper presented at the Transportation Research Board 99th Annual Meeting, Washington, D.C., USA. 
Delmelle, E., \& Casas, I. (2012). Evaluating spatial equity of bus rapid transit-based accessibility patterns in a developing country: The case of Cali, Colombia. Transport Policy, 20, 36 - 46

El-Geneidy, A., \& Levinson, D. (2006). Access to destinations: Development of accessibility measures. Retrieved from St-Paul, Minnesota, US: http://nexus.umn.edu/projects/Access/AccessFinalReport.pdf

El-Geneidy, A., \& Levinson, D. (2011). Place Rank: Valuing spatial interactions. Networks and Spatial Economics (NETS), 11(4), 643-659.

El-Geneidy, A., Levinson, D., Diab, E., Boisjoly, G., Verbich, D., \& Loong, C. (2016). The cost of equity: Assessing transit accessibility and social disparity using total travel cost. Transportation Research Part A, 302-316.

Foth, N., Manaugh, K., \& El-Geneidy, A. (2013). Towards equitable transit: Examining transit accessibility and social need in Toronto, Canada 1996-2006. Journal of Transport Geography, 29, 1 - 10.

Geurs, K., \& van Wee, B. (2004). Accessibility evaluation of land-use and transport strategies: Review and research directions. Journal of Transport Geography, 12(2), 127-140.

Golub, A., \& Martens, K. (2014). Using principles of justice to assess the modal equity of regional transportation plans. Journal of Transport Geography, 41, 10-20.

Grengs, J. (2015). Nonwork accessibility as a social equity indicator. International Journal of Sustainable Transportation, 9(1), 1 - 14.

Guagliardo, M. F. (2004). Spatial accessibility of primary care: concepts, methods and challenges. International Journal of Health Geographics, 3(3).

Guagliardo, M. F., Ronzio, C. R., Cheung, I., Chacko, E., \& Joseph, J. G. (2004). Physician accessibility: an urban case study of pediatric providers. Health \& place, 10(3), $273-283$.

Guzman, L., Oviedo, D., \& Rivera, C. (2017). Assessing equity in transport accessibility to work and study: The Bogotá region. Journal of Transport Geography, 58, 236 - 246.

Handy, S., \& Niemeier, D. (1997). Measuring accessibility: an exploration of issues and alternatives. Environment and planning A, 29(7), 1175 - 1194.

Hansen, W. (1959). How accessibility shapes land use. Journal of the American Institute of Planners, 25(2), 73-76.

Haynes, R., Lovett, A., \& Sunnenberg, G. (2003). Potential accessibility, travel time, and consumer choice: Geographical variations in general medical practice registration in Eastern England. Environment and Planning A, 35, 1733 - 1750.

Health Canada. (2017). Canada Health Act: 2015 - 2016 annual report. Retrieved from https://www.canada.ca/content/dam/hc-sc/documents/services/publications/health-systemservices/canada-health-act-annual-report-2015-2016.pdf

Higgs, G., Zahnow, R., Corcoran, J., Langford, M., \& Fry, R. (2017). Modelling spatial access to General Practitioner surgeries: Does public transport availability matter? Journal of Transport \& Health, 6, 143-154. doi:https://doi.org/10.1016/j.jth.2017.05.361

Hiscock, R., Pearce, J., Blakely, T., \& Witten, K. (2008). Is neighbourhood access to health care provision associated with individual-level utilization and satisfaction? Health Services Research, 43, 2183 2200.

Hochstenbach, C., \& Musterd, S. (2017). Gentrification and the suburbanization of poverty: Changing urban geographies through boom and bust periods. Urban Geography, 39(1), 26-53.

Joseph, A. E., \& Bantock, P. R. (1982). Measuring potential physical accessibility to general practitioners in rural areas: A method and case study. Social Science and Medicine, 16, 85 - 90.

Joseph, A. E., \& Phillips, D. R. (1984). Accessibility and utilization: Geographical perspectives on health care delivery. New York: Harper and Row Publishers.

Keeling, D. (2008). Latin America's transportation conundrum. Journal of Latin American Geography, 133154. 
Kneebone, E. (2017). The changing geography of US poverty. Retrieved from https://www.brookings.edu/testimonies/the-changing-geography-of-us-poverty/

Korda, R. J., Butler, J. R., Clements, M. S., \& Kunitz, S. J. (2007). Differential impacts of health care in Australia: Trend analysis of socioeconomic inequalities in avoidable mortality. International Journal of Epidemiology, 36, 157 - 165.

Korsu, E., \& Wenglenski, S. (2010). Job accessibility, residential segregation, and risk of long-term unemployment in the Paris region. Urban Studies, 47(11), 2279 - 2324.

Law, M., Dijkstra, A., Douillard, J., \& Morgan, S. (2011). Geographic accessibility of community pharmacies in Ontario. Healthcare Policy, 6(3), 36.

Love, D., \& Lindquist, P. (1995). The geographical accessibility of hospitals to the aged: a geographic information systems analysis within Illinois. Health Services Research, 29(6), 629 - 651

Lucas, K., van Wee, B., \& Maat, K. (2016). A method to evaluate equitable accessibility: Combining ethical theories and accessibility-based approaches. Transportation, 43(3), 473-490.

Luo, W., \& Wang, F. (2003). Measures of spatial accessibility to health care in a GIS environment: synthesis and a case study in the Chicago region. Environment and Planning B: Planning and Design, 30, 865 $-884$.

Mao, L., \& Nekorchuk, D. (2013). Measuring spatial accessibility to healthcare for populations with multiple transportation modes. Health \& place, 24, 115 - 122.

Martin, D., Jordan, H., \& Roderick, P. (2008). Taking the bus: incorporating public transport timetable data into health care accessibility modelling. Environment and Planning A, 4O(10), 2510-2525.

McGrail, M. R., \& Humphreys, J. S. (2009). Measuring spatial accessibility to primary care in rural areas: Improving the effectiveness of the two-step floating catchment area method. Applied Geography, $29,533-541$.

Mortazavi, S., \& Akbarzadeh, M. (2017). A framework for measuring the spatial equity in the distribution of public transportation benefits. Journal of Public Transportation, 20(1), 44 - 62.

Mueller, N., Rojas-Rueda, D., Basagaña, X., Cirach, M., Cole-Hunter, T., Dadvand, P., . . Nieuwenhuijsen, M. (2017). Health impacts related to urban and transport planning: A burden of disease assessment. Environment International, 243-257. doi:https://doi.org/10.1016/j.envint.2017.07.020

Neutens, T. (2015). Accessibility, equity and health care: Review and research directions for transport geographers. Journal of Transport Geography, 43, 14 - 27.

Paez, A., Mercado, R., Farber, S., Morency, C., \& Roorda, M. (2010). Accessibility to health care facilities in Montreal Island: an application of relative accessibility indicators from the perspective of senior and non-senior residents. International Journal of Health Geographics, 9(52), 1-15.

Paez, A., Ruben, M., Faber, S., Morency, C., \& Roorda, M. (2009). Mobility and Social Exclusion in Canadian Communities. Retrieved from https://www.researchgate.net/profile/Catherine Morency/publication/233997689 Mobility an d Social Exclusion in Canadian Communities An Empirical Investigation of Opportunity Acc ess and Deprivation/links/54f066980cf25f74d7267b6f/Mobility-and-Social-Exclusion-inCanadian-Communities-An-Empirical-Investigation-of-Opportunity-Access-and-Deprivation.pdf

Paez, A., Scott, D., \& Morency, C. (2012). Measuring accessibility: Positive and normative implementations of various accessibility indicators. Journal of Transport Geography, 25, 141-153.

Parliament of Canada. (1985). Canada Health Act. Ottawa, Canada Retrieved from http://lawslois.justice.gc.ca/PDF/C-6.pdf

Pavlic, D., \& Qian, Z. (2014). Declining inner suburbs? A longitudinal-spatial analysis of large metropolitan regions in Canada. Urban Geography, 35(3), 378-401.

Pereira, R., Schwanen, T., \& Banister, D. (2017). Distributive justice and equity in transportation. Transport Reviews, 37(2), 170-191. 
Raphael, S., \& Stoll, R. (2010). Job sprawl and the suburbanization of poverty. Retrieved from Washington DC: https://gspp.berkeley.edu/assets/uploads/research/pdf/p60.pdf

Schoner, J., Chapman, J., Brookes, A., MacLeod, K., Fox, E., Iroz-Elardo, N., \& Frank, L. (2018). Bringing health into transportation and land use scenario planning: Creating a National Public Health Assessment Model (N-PHAM). Journal of Transport \& Health, 10, 401-418. doi:https://doi.org/10.1016/j.jth.2018.04.008

Shen, Q. (1998). Location characteristics of inner-city neighborhoods and employment accessibility of lowwage workers. Environment and Planning B: Planning and Design, 25, 345 - 365.

Statistics Canada. (2016). Statistics Canada Census 2016.

Syed, S., Gerber, B., \& Sharp, L. (2013). Traveling towards disease: Transportation barriers to health care access. Journal of Community Health, 38(5), 976-993.

Tang, J., Chiu, Y., Chiang, P., Su, M., \& Chan, T. (2017). A flow-based statistical model integrating spatial and nonspatial dimensions to measure healthcare access. Health \& place, 47, $126-138$.

van Wee, B., \& Geurs, K. (2011). Discussing equity and social exclusion in accessibility evaluations. European Journal of Transport and Infrastructure Research, 11(4).

Weibull, J. (1976). An axiomatic approach to the measurement of accessibility. Regional Science and Urban Economics, 6, 357 - 379.

Wickstrom, G. (1971). Defining balanced transportation: A question of opprtunity. Traffic Quarterly, 25(3), 337-349.

Wilson, K., \& Rosenberg, M. W. (2004). Accessibility and the Canadian health care system: Squaring perceptions and realities. Health Policy, 67, 137 - 148. 